\title{
Inhaltsübersicht - Band II
}

\begin{tabular}{|c|c|c|c|c|c|c|c|c|c|c|c|c|}
\hline & & & & & & Seite & & & & & & Seite \\
\hline Inula Helenium & . & . & & . & & 1 & Petroselinum . & & . & & & 104 \\
\hline ris . . . . & . & . & & . & & 2 & Peucedanum . & & . & . & & 107 \\
\hline & & & & & & & Phaseolus . . . & & . & . & . & 110 \\
\hline Juglans . & . & . & . & . & & 4 & Pimpinella Anisum & & & & & 112 \\
\hline Juniperus . . & . & . & - & . & . & 8 & Pimpinella saxifraga & & nd & & & \\
\hline Juniperus Sabir & a. & . & . & . & & 13 & Pimpinella magna. & & . & . & . & \\
\hline Juniperus Oxyce & edrus & . & . & . & & 15 & Pinus. . . . . & & . & . & . & \\
\hline aminaria & . & . & . & . & . & 16 & Pirus aucuparia. . & & . & . & , & \\
\hline amium . & . & . & . & . & & 17 & Pistacia . . . . & & . & . & . & 153 \\
\hline arix . . & . & . & . & . & . & . & Plantago . . . & & . & . & . & 156 \\
\hline Laurus . & . & t & . & . & & & Polygala & & . & . & . & \\
\hline Lavandula . & . & . & . & . & & 24 & Polygonum & & . & . & . & \\
\hline Ledum palustre & . & . & . & . & . & 28 & Polypodium . . . & & . & . & . & 166 \\
\hline urus . . & . & . & . & . & . & . & Polyporus . . . & & . & . & . & \\
\hline Levisticum . & . & . & . & . & . & & Populus . & & . & . & . & \\
\hline Linaria ... & . & . & . & . & & . & Potentilla . . . & & . & . & . & \\
\hline Linum . & . & . & . & . & & 36 & Primula . . . . & & . & . & . & \\
\hline Cobelia . . & 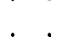 & r & . & . & & 38 & Prunus Amygdalus. & & . & . & . & \\
\hline Lycopodium & & & . & 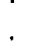 & & 42 & Prunus avium . . & & - & . & . & \\
\hline & & & & & & & Prunus cerasus . . & & . & . & . & \\
\hline Malva . . & . & . & . & . & . & 45 & Prunus domestica . & & . & . & . & 188 \\
\hline Marrubium & . & . & . & . & . & 49 & Prunus Laurocerasus & & . & . & . & \\
\hline Matricaria . & . & . & . & . & . & 50 & Prunus padus . . & & . & . & . & \\
\hline Melilotus . & . & . & . & . & . & . & Prunus persica und & & & & & \\
\hline Melissa . & . & . & . & . & . & 57 & Prunus armeniaca. & & . & . & . & 191 \\
\hline Mentha . . & . & . & . & . & & 59 & Prunus spinosa . . & & . & . & . & \\
\hline Menyanthes & . & . & . & . & . & 70 & Prunus virginiana . & & . & . & . & \\
\hline Nigella . & - & . & - & . & ${ }^{\circ}$ & 71 & $\begin{array}{l}\text { Pulmonaria . . } \\
\text { Punica granatum . }\end{array}$ & & · & . & $\cdot$ & . \\
\hline the aquat & & • & . & . & & & Quercus & & . & . & & \\
\hline Olea europaea & . & . & . & . & & 75 & mnus Frangula. & & & & & \\
\hline Ononis spinosa & & . & . & · & & 78 & $\begin{array}{l}\text { Rhamnus Frangula } \\
\text { Rhamnus cathartica }\end{array}$ & & $\bullet$ & $\cdot$ & $\cdot$ & $\begin{array}{l}\cdot 201 \\
\cdot \quad 210\end{array}$ \\
\hline Orchis . . & $\cdot \operatorname{rana}$ & & - & - & & 79 & Rheum . . . . & & . & . & • & \\
\hline Origanum Major & rana & & $\cdot$ & $\cdot$ & & 83 & Ribes nigrum . . & & . & . & . & \\
\hline Origanum vulga & & & $\cdot$ & $\cdot$ & & 88 & Ribes rubrum . & & $\cdot$ & & & \\
\hline Origanum cretic & $\operatorname{cum}$ & & $\cdot$ & . & & & Ricinus communis . & & & & & \\
\hline Paeonia . . . & & 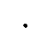 & . & . & . & 89 & Rorippa Nasturtium & & & ticu & um & \\
\hline Papaver Rhoeas & & & . & . & • & 91 & Rosa . . . . . & & . & . & . & 230 \\
\hline Papaver somnife & erum & & . & & $\cdot$ & 93 & Rosa canina . . & & . & . & . & . 235 \\
\hline
\end{tabular}




\begin{tabular}{|c|c|c|c|c|c|c|c|c|}
\hline & & & & Seite & & & & Seite \\
\hline osmarinus . & & & • & 237 & Tilia . . & & & 29 \\
\hline Rubus idaeus . & & & • & 241 & Trigonella . & & . & \\
\hline Rubus fructicosus & ${ }^{\circ}$ & & & 243 & Triticum & & . & 29 \\
\hline uta . . . . . & & & . & 245 & Tussilago Farfara . & & . & \\
\hline alix . . . & . & . & . & 248 & Urginea maritima . & & . & \\
\hline Salvia . . . & . & . & . & 250 & Urtica . . . . & & . & \\
\hline Salvia sclarea . & . & & . & 254 & & & & \\
\hline Sambucus nigra. & & & & 254 & Vaccinium myrtillus . & & - & \\
\hline ambucus ebulus & & • & & 259 & Vaccinium Vitis idaea & & · & \\
\hline Sambucus racemosa & . & & . & 261 & Valeriana . . . . & & $\cdot$ & \\
\hline Saponaria . . . & & . & . & 262 & Veratrum. & & . & \\
\hline Satureja . & & & . & 264 & Verbascum • . . & & · & \\
\hline Secale cornutum & & & & 265 & Verbena $\cdot \cdot \cdot \cdot$ & & . & \\
\hline Silybum Marianum & & & & 269 & Veronica . . . . . & & $\cdot \cdot$ & \\
\hline Sinapis alba ... & & & • & 270 & Viola odorata $\cdot$. & & $\cdot$ & $\cdot$ \\
\hline Solanum dulcamara & & & & . 272 & Viola tricolor. & & - & \\
\hline Solanum nigrum & & & & . 274 & Viscum album & & . $\cdot$ & \\
\hline Solanum tuberosum & . & . & . & . 275 & Zea Mays. & & $\cdot$ & \\
\hline m-Arten & & & & . 276 & & & & \\
\hline Solidago Virgaurea & & & & . 277 & $\begin{array}{l}\text { Gesamt-Register der la } \\
\text { Drogen- und Stam }\end{array}$ & & $\begin{array}{l}\text { inischen } \\
\text { flanzen- }\end{array}$ & \\
\hline Taraxacum & ( & & & . 279 & namen.... & & $\cdot \cdot$ & \\
\hline $\begin{array}{l}\text { Thuja } \\
\text { Thymus vulgaris }\end{array}$ & & & - & $\begin{array}{l}281 \\
\text {. } 284\end{array}$ & $\begin{array}{l}\text { egister der } \\
\text { emdsprachigen }\end{array}$ & & andel & \\
\hline Thymus Serpyllum & & & & . 289 & bezeichnungen . & & . . & \\
\hline
\end{tabular}




\section{Inhaltsübersicht - Band I}

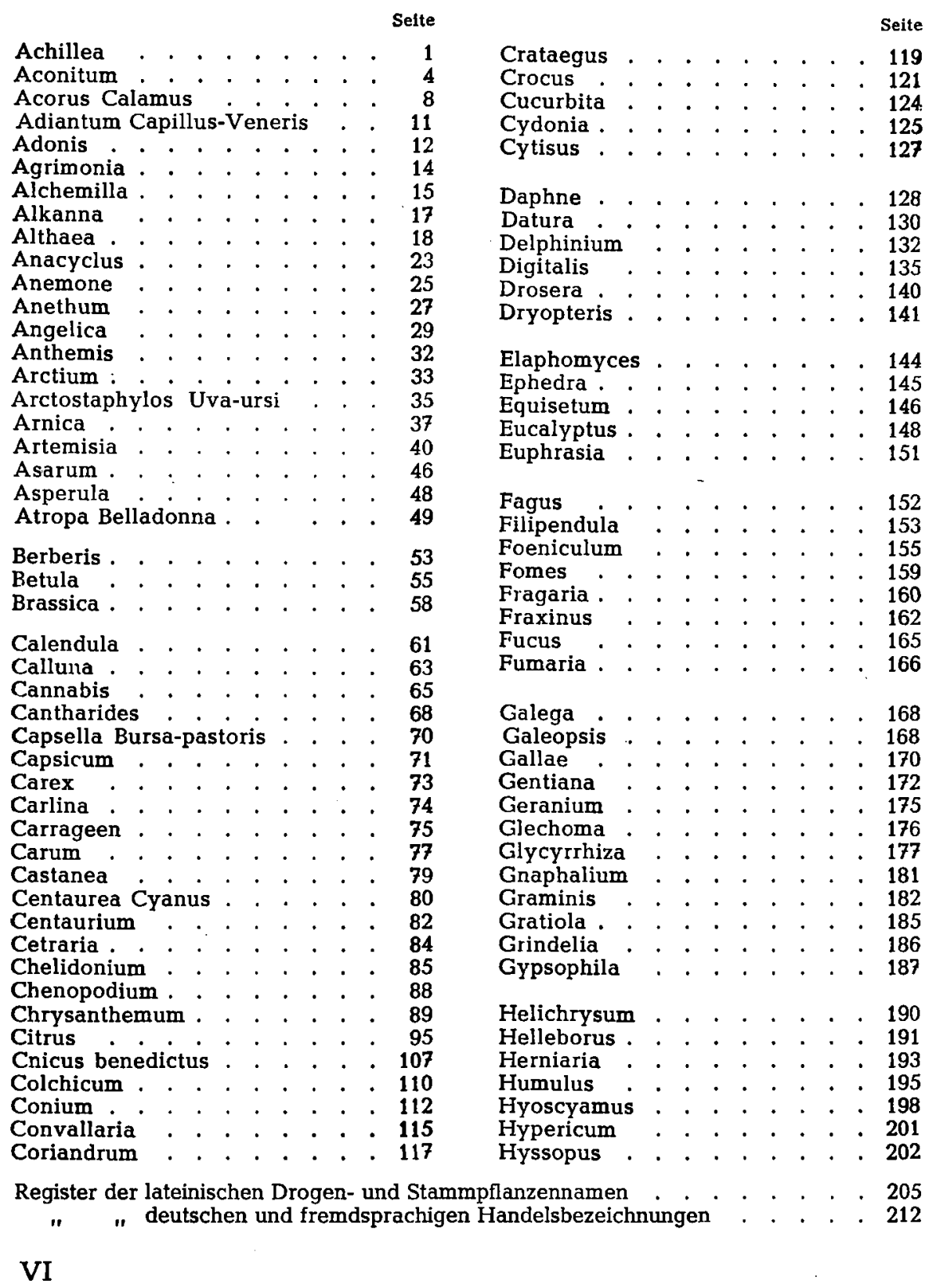

